\title{
IMC Y GRASA CORPORAL DE NIÑOS DE COLEGIOS MUNICIPALIZADOS Y PARTICULARES DE VIÑA DE MAR
}

\author{
BMI AND BODY FAT IN CHILDREN OF MUNICIPAL AND PARTICULAR VIÑA DEL MAR \\ COLLEGES
}

\author{
Allende Paredes, Paola; Carvajal Cortés, María Francisca; Puebla Vera, Robinson \& Rodríguez Rodríguez, \\ Fernando.
}

Escuela de Educación Física, Pontificia Universidad Católica de Valparaíso, Chile.

\begin{abstract}
ALLENDE P.; CARVAJAL MF.; PUEBLA R. \& RODRÍGUEZ F. IMC y grasa corporal de niños de colegios municipalizados y particulares de Viña del Mar. Rev. Motr. Hum., 11(1): 20-23, 2010.

RESUMEN

Las evaluaciones antropométricas son una herramienta útil, de bajo costo y fácil de usar para la determinación del estado nutricional de las personas. Los sujetos evaluados son niños y niñas de $5^{\circ}$ y $6^{\circ}$ básico, con edades de entre 10 y 12 años, pertenecientes a establecimientos educacionales de la ciudad de Viña del Mar. Se diseña un protocolo de evaluación antropométrica de acuerdo a la edad de los estudiantes, midiendo el peso corporal y la talla corporal para determinar el Índice de Masa Corporal (IMC), y seis pliegues cutáneos para la estimación de la masa grasa. Es importante considerar que el IMC de ambos grupos se encuentra entre el percentil 75 (p75) y el percentil 85 (p85), considerado normal pero en un nivel alto, ya que el IMC normal se encuentra en el rango de percentiles 5 y 85. Adicional al aporte del IMC usamos la cuantificación de la grasa corporal como un factor determinante en la salud de los escolares. Futuros estudios deberían considerar la inclusión de la masa grasa como índice de salud, así como la construcción de referencias locales y actualizadas de peso, talla e IMC para la población chilena.
\end{abstract}

Palabras Clave: IMC, grasa corporal, estado nutricional.

\section{INTRODUCCIÓN}

Las evaluaciones antropométricas son una herramienta útil, de bajo costo y fácil de usar para la determinación del estado nutricional de las personas (1).

El diagnóstico nutricional que entrega la antropometría permite tener una idea del riesgo nutricional por mal nutrición por exceso o mal nutrición por déficit. Esto permite crear políticas públicas para mejorar los problemas relacionados con la alimentación y nutrición.

El IMC es un índice de baja confiabilidad (2), pero usado en todo el mundo para clasificar los estados nutricionales de bajo peso, normopeso, sobrepeso, etcétera (3). Este índice no discrimina la composición corporal que puede aumentar el peso corporal, obteniendo en consecuencia un IMC más alto, sin considerar que este peso puede estar aumentado por la masa muscular predominante y el tejido óseo denso.

Es una inquietud conocer las diferencias en la estructura corporal de los escolares y sus diferencias en el actual sistema educativo. Éstas se ven reflejadas, principalmente, en el hecho que nos enfrentamos a dos realidades totalmente distintas: por un lado encontramos la realidad de un colegio particular, en el que parte de los hábitos alimenticios vienen desde el hogar y se entrega también la posibilidad de parte del colegio para alimentarse, por lo que entre los alumnos no existen mayores distancias. En cambio, en un colegio municipal la realidad es otra, ya que el colegio no tiene la oportunidad de brindarles a todos los alumnos las mismas oportunidades de alimentación, y también los hábitos desde el hogar no son siempre los ideales, condiciones que pueden alterar la morfoestructura de los alumnos. Intentaremos resolver nuestra pregunta evaluando estas variables en ambos tipos de establecimientos educativos.

\section{METODOLOGÍA}

Nuestro estudio es del tipo Descriptivo - Exploratorio, no experimental y transversal; es decir, "miden, evalúan y recolectan los datos sobre diversos aspectos, dimensiones o componentes del fenómeno a investigar" (4), con el fin de recolectar toda la información necesaria para llegar al resultado de nuestra investigación.

También se considera Cuantitativo, porque "analiza diversos elementos que pueden ser medidos y cuantificados. Toda la información se obtiene a base de muestras de la población, y sus resultados son extrapolables a toda la población, con un determinado nivel de error y nivel de confianza. Ésta, se sirve de números y métodos estadísticos. Parte de casos concretos para llegar a una general o comprobar causales. Se dice cuantitativa- sistemática- generalizadora" (5). Los sujetos evaluados son niños y niñas de $5^{\circ}$ y $6^{\circ}$ básico, con edades de entre 10 y 12 años, pertenecientes a establecimientos educacionales de la ciudad de Viña del Mar. De ellos, dos son municipales: uno mixto -Escuela 
ALLENDE P.; CARVAJAL MF.; PUEBLA R. \& RODRÍGUEZ F. IMC y grasa corporal de niños de colegios municipalizados y particulares de Viña del Mar. Rev. Motr. Hum., 11(1): 20-23, 2010.

Santa María- y el otro femenino -Escuela República del Ecuador-; y dos colegios particulares: uno mixto, Colegio Saint Dominic; y el otro masculino, The Mackay School. La muestra escogida intencionalmente la componen 192 alumnos, los cuales 102 pertenecen a establecimientos municipales y 90 a establecimientos particulares. Se diseña un protocolo de evaluación antropométrica de acuerdo a la edad de los estudiantes, midiendo el peso corporal y la talla corporal para determinar el Índice de Masa Corporal (IMC) y seis pliegues cutáneos para la estimación de la masa grasa.

\begin{tabular}{cc} 
Categorías & Rango en Percentiles \\
\hline Bajo Peso & $<$ percentil 5 \\
Normal & Entre percentil 5 y 85 \\
Sobrepeso & Entre percentil 85 y 95 \\
Obesidad & $>$ percentil 95
\end{tabular}

Tabla 1: Categorías del IMC para la edad del Center for Disease Control and Prevention (CDC), EEUU, 2000.
La cuantificación y clasificación del nivel nutricional del IMC se realiza con el uso de percentiles (Tabla 1). Los pliegues cutáneos evaluados son: Subescapular, Tricipital, Suprailíaco, Abdominal, Muslo y Pierna. Se decide usar estos pliegues ya que consideran la estimación de la grasa del miembro inferior, utilizando la fórmula de Deborah Kerr (6).

\section{RESULTADOS}

Se valoran los dos componentes antropométricos básicos en ambos tipos de establecimientos educativos y separados por sexo, encontrando diferencias sólo en la talla corporal de las alumnas y diferencias en el peso corporal y talla corporal de los alumnos, comparando los dos tipos de establecimientos (Tabla 2).

\begin{tabular}{ccccc} 
Tipo de establecimiento & \multicolumn{2}{c}{ Femenino } & \multicolumn{2}{c}{ Masculino } \\
\hline & PESO & TALLA & PESO & TALLA \\
Municipal & 41,7 & 143,7 & 38,8 & 144,8 \\
Particular & 44,7 & $150,1\left(^{*}\right)$ & 46,0 & $151,3(*)$
\end{tabular}

Tabla 2: Resumen descriptivo de las diferencias en el peso y talla de los alumnos de ambos tipos de establecimientos (*) Diferencias significativas para $\mathrm{p}<0,05$ en una Prueba $T$.

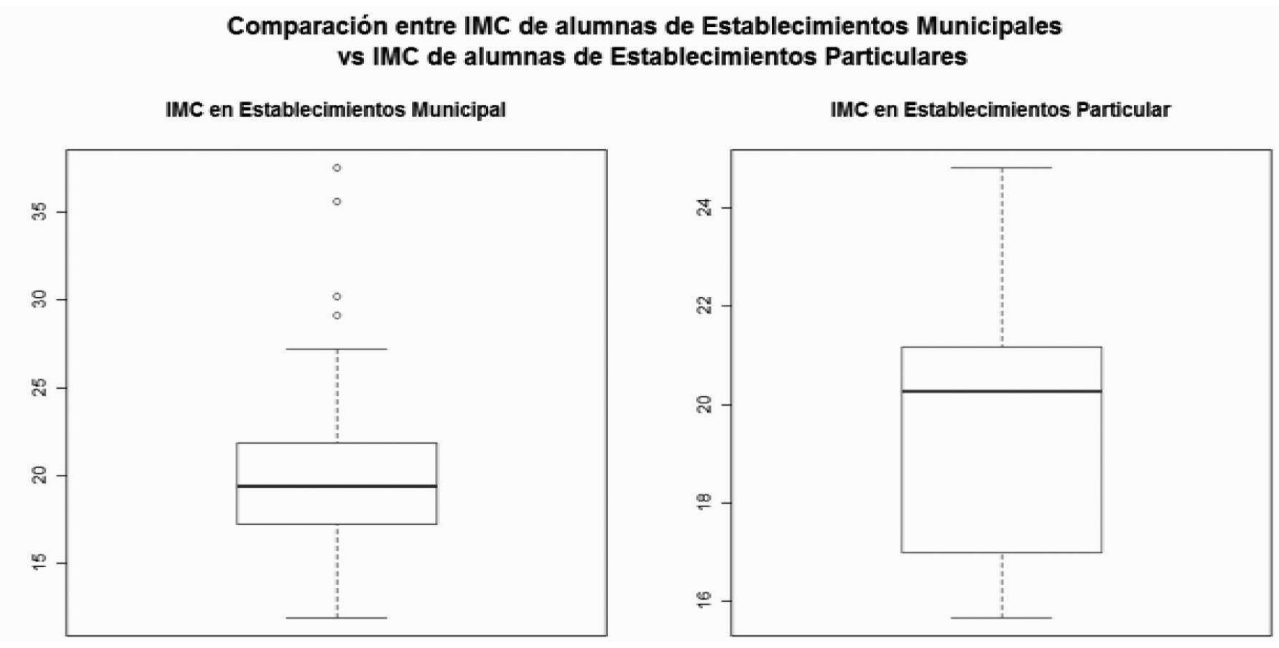

Figura 1: Comparación entre IMC de alumnas de Establecimientos Municipales v/s alumnas de Establecimientos Particulares.

Con el propósito de verificar esta diferencia se realizó la prueba no paramétrica de Kruskal Wallis, obteniéndose chisquared $=0, d f=1, p$-value $=0.97$.

Dado un nivel de significación mayor al 5\%, podemos concluir que no existen diferencias significativas entre el IMC de Establecimientos Municipales versus IMC de Establecimientos Particulares.

Sin embargo, es importante destacar que aunque no existan 
Allende P.; CARVAJAL MF.; PUEbla R. \& RODRÍGUEZ F. IMC y grasa corporal de niños de colegios municipalizados y particulares de Viña del Mar. Rev. Motr. Hum., 11(1): 20-23, 2010.

diferencias significativas, encontramos que en los Establecimientos Municipales existen grupos de alumnas que presentan sobrepeso y una minoría, obesidad; mientras ésta última no se aprecia en los Establecimientos Particulares. Es importante destacar que, aunque no existan diferencias significativas en la masa adiposa de los alumnos de los Establecimientos Municipales versus los alumnos de los Establecimientos Particulares al analizar los datos de cada pliegue; individualmente podemos visualizar diferencias entre un establecimiento y otro.

\begin{tabular}{lcccccc} 
& \multicolumn{3}{c}{ Femenino } & \multicolumn{3}{c}{ Masculino } \\
\cline { 2 - 7 } Municipal & IMC & SUM PL & M ADIP & IMC & SUM PL & M ADIP \\
Particular & 19,2 & 138,0 & 20,5 & 18,5 & 103,6 & 16,8 \\
& 19,9 & $110,0\left(*^{*}\right)$ & $18,4\left(^{*}\right)$ & 20,1 & 100,7 & 17,0
\end{tabular}

Tabla 3: Resumen descriptivo de las diferencias en el IMC, Sumatoria de pliegues (SUM PL) Y Masa Adiposa en \% (M ADIP), de los alumnos de ambos tipos de establecimientos.

(*) Diferencias significativas para $\mathrm{p}<0,05$ en una Prueba $\mathrm{T}$.

\section{DISCUSIÓN}

En los Establecimientos Municipales se presenta un promedio de peso de $38,8 \mathrm{~kg}$; mientras que en los Establecimientos Particulares es de 46,0 kg. Por lo tanto, se puede apreciar una diferencia de $7 \mathrm{~kg}$.

Respecto al indicador Talla, los Establecimientos Municipales presentan un promedio de 144,8 cm; mientras que en los Establecimientos Particulares es de 151,1 cm. Con esto, podemos inferir que los Establecimientos Particulares tienen mayor peso debido a que presentan una mayor talla. Por el contrario, los Establecimientos Municipales presentan un menor peso, pudiendo observarse que son más delgados; sin embargo, las características que poseen respecto a su altura nos indican que se encuentran dentro de los parámetros adecuados a su edad (7). Podemos determinar que no existen diferencias significativas en el Índice de Masa Corporal (IMC) entre ambos tipos de Establecimientos Educacionales, según el test KruskalWallis, que dio como resultado p-valué $=\mathbf{0 . 2 3 1 5}$; ya que tiene un nivel de significación mayor al 5\%. Esto, a partir que los varones de los colegios Municipales tienen un IMC promedio de $19,88 \mathrm{~kg} / \mathrm{m}^{2}$; mientras que los varones de $10 \mathrm{~s}$ colegios Particulares tienen un promedio de $20,03 \mathrm{~kg} / \mathrm{m}^{2}$. Sin embargo, podemos encontrar las diferencias en los indicadores Peso y Talla por separado.

En el caso de las mujeres tampoco se encontraron grandes diferencias en el Índice de Masa Corporal, teniendo los Establecimientos Municipales un promedio de 20,03 kg y los Establecimientos Particulares un 19,66 kg; según el test de Kruskal-Wallis esta diferencia no es significativa, ya que dio un resultado de $\mathbf{p}$-valué $\mathbf{= 0 . 9 7}$.

Es importante considerar que el IMC de ambos grupos se encuentra entre el percentil 75 (p75) y el percentil 85 (p85), considerado normal; pero en un nivel alto, ya que el IMC normal se encuentra en el rango de percentiles 5 y 85 (8). Idealmente, se recomienda clasificar el estado nutricional por IMC en relación a los estadios de maduración de Tanner
(9) y considerando el dismorfismo sexual entre hombres y mujeres de esta edad (10).

Al igual que en los varones, se destacan las diferencias existentes en cada uno de los indicadores (Peso y Talla). En el Peso, las diferencias son las siguientes: en los Establecimientos Municipales, el promedio obtenido es de 41, $7 \mathrm{~kg}$; en cambio, en los Establecimientos Particulares es $44,7 \mathrm{~kg}$. La diferencia es de $3 \mathrm{Kg}$. Asimismo, en la Talla también existe una diferencia: en los Establecimientos Municipales presentan una estatura promedio de 143, $7 \mathrm{~cm}$; mientras que en los Establecimientos Particulares promedian una estatura de $150,1 \mathrm{~cm}$. Es decir, existe una diferencia de aproximadamente $6 \mathrm{~cm}$.

Una explicación a estas diferencias significativas en las variables Peso y Talla (Tabla 2), tanto para hombres como para mujeres entre los Establecimientos Particulares y Municipales, es la posible ascendencia extranjera (Europea y Americana) que caracteriza a los escolares de colegios Particulares, teniendo factores genéticos que influyen netamente en las características físicas, en este caso la Estatura y el Peso (11).

Adicional al aporte del IMC, usamos la cuantificación de la grasa corporal como un factor determinante en la salud de los escolares (12).

Los resultados demuestran que sólo hay diferencias estadísticas en la cantidad de grasa en las mujeres de ambos tipos de establecimiento, donde es más elevado en las niñas de colegios municipalizados; pero no hay diferencias estadísticas en los hombres, pese a ser levemente más alto en hombres de colegios municipales.

Esta diferencia en las mujeres puede deberse a factores socioeconómicos, donde se estima que la calidad de la alimentación es mejor en hogares de niveles económicos más altos, considerando que a esta edad las niñas tienen un estado de maduración similar (13).

Los resultados de masa grasa pueden interpretarse 
ALLENDE P.; CARVAJAL MF.; PUEbla R. \& RODRÍGUEZ F. IMC y grasa corporal de niños de colegios municipalizados y particulares de Viña del Mar. Rev. Motr. Hum., 11(1): 20-23, 2010.

distintamente en los hombres, ya que pueden encontrarse en estados madurativos diferentes.

Las cantidades de masa grasa de los escolares se encuentran aún disminuidas, sobre todo en varones, lo que explica de mejor manera el estado de maduración más bajo de escolares mujeres (Tabla 3).

Futuros estudios deberían considerar la inclusión de la masa grasa como índice de salud, así como la construcción de referencias locales y actualizadas de peso, talla e IMC para la población chilena.

\section{BIBLIOGRAFÍA}

1. Alarcón M \& Atalah E. Confiabilidad de las mediciones antropométricas en escolares de la comuna de Vicuña (Chile). Rev. chil. nutr., vol.36. 2009; 4; 1056-1062.

2. Gallagher, D, Visser M, Sepúlveda D, Pierson R, Harris $\mathrm{T}$ \& Heymsfield S. How useful is body mass index for comparison of body fatness across age, sex, and ethnic groups? Am. J. Epidemiol. 1996. 143: 228-239.

3. Van Itallie T \& Abraham S. Some hazards of obesity and its treament. In Hirsch J \& Van Itallie T (eds). Recent Advances in Obesity Research IV. London: J. Libbey, 1985. pp 1-19.

4. Hernández y cols. Metodología de la Investigación. Cap. 3 p.117. 2003.

5. Mendoza R. Investigación Cualitativa y Cuantitativa. Diferencias y Limitaciones. 2006.
6. Kerr D. An anthropometric method for the fractionation of skin, adipose, muscle, bone and residual tissue masses in males and females age 6 to 77 years. M. Sc. Thesis. Simon Fraser University. 1988.

7. Macmillan N. Nutrición Deportiva. Ediciones Universitarias. 2006.

8. Cole T, Bellizi K, Flegal M, Dietz W. Body mass index in children worldwide: cut off points for overweight and obesity. BMJ 2000. 320: 1-6.

9. Burrows R, Díaz N \& Muzzo S. Variaciones del índice de masa corporal (IMC) de acuerdo al grado de desarrollo puberal alcanzado. Rev. méd. Chile. 2004, vol.132, n.11

10. Almagiá A et al. Estudio y análisis morfológico etario del dimorfismo sexual a través de la composición corporal. Rev. Chil. Anat.1997, vol.15, n.2, pp. 141-149.

11. Burrows R, Leiva L, Zvaigafth A, Muzzo S. Influencia del NSE en la composición corporal y estatura de escolares durante la pubertad. Rev. Chil. Nutr. 1989; 17: 39-45.

12. Cole T, Faith M, Pietrobelli A \& Heo M. What is the best measure of adiposity change in growing children: BMI, $B M I \%$, BMI z-score or BMI centile? European Journal of Clinical Nutrition. 2005. 59, 419-425.

13. Bundred P, Kitchiner D, Buchan I. Prevalence of overweight and obese children between 1989-1998: population based series of cross sectional studies. BMJ 2001. 322: bmj.com,1-3.

\begin{abstract}
Anthropometric assessments are a useful, inexpensive and easy to use for determining the nutritional status of individuals. The subjects evaluated were children of 5th and 6th grade, aged between 10 and 12 years, belonging to educational institutions in the city of Viña del Mar. We designed an anthropometric assessment protocol according to the age of students, measuring body weight and height to determine body mass index (BMI) and six skinfolds to estimate fat mass. It is important to note that the BMI of both groups is between the 75th percentile ( $p 75)$ and the 85th percentile ( $p 85)$, considered normal, but at a high level as the normal BMI is in the range of 5 and 85 percentiles. In addition to the contribution of BMI, we used the quantification of body fat as a factor in the health of schoolchildren. Future studies should consider including fat mass as an index of health as well as the construction of local references and current weight, height and BMI for the Chilean population.
\end{abstract}

Key words: BMI, fat mass, nutritional state.

\title{
Dirigir correspondencia a:
}

Fernando Rodríguez Rodríguez

Avenida El Bosque 1290, Santa Inés, Viña del Mar

CHILE

Fono: (56) 322274381

E-mail: fernando.rodriguez@ucv.cl 